\title{
A COMPLETE SOLUTION FOR THE OPTIMAL STOCHASTIC SCHEDULING OF A TWO-STAGE TANDEM QUEUE WITH TWO FLEXIBLE SERVERS
}

\author{
KLAUS SCHIEFERMAYR, ${ }^{*}$ Upper Austrian University of Applied Sciences, Wels \\ JOSEF WEICHBOLD, ${ }^{* *}$ Universität Linz
}

\begin{abstract}
We consider a two-stage tandem queue with two parallel servers and two queues. We assume that initially all jobs are present and that no further arrivals take place at any time. The two servers are identical and can serve both types of job. The processing times are exponentially distributed. After being served, a job of queue 1 joins queue 2, whereas a job of queue 2 leaves the system. Holding costs per job and per unit time are incurred if there are jobs holding in the system. Our goal is to find the optimal strategy that minimizes the expected total holding costs until the system is cleared. We give a complete solution for the optimal control of all possible parameters (costs and service times), especially for those parameter regions in which the optimal control depends on how many jobs are present in the two queues.
\end{abstract}

Keywords: Optimal stochastic scheduling; flexible server; tandem queue; clearing system

2000 Mathematics Subject Classification: Primary 90E20

Secondary 90B22; 60K25

\section{Introduction}

We consider a two-stage tandem queue with two parallel servers and two queues, queue 1 and queue 2 . In the system, there are initially $n_{1}$ jobs at queue 1 and $n_{2}$ jobs at queue 2 , and there are no further arrivals at any later time. The two servers are identical and can serve both types of jobs. The processing time of a job at queue 1 or queue 2 is exponentially distributed with mean $1 / \mu_{1}$ or $1 / \mu_{2}$, respectively. After being processed, a job of queue 1 joins queue 2 , whereas a job of queue 2 that has been served leaves the system. A holding $\operatorname{cost} c_{i}, i=1,2$, per job and per unit time is incurred if there are jobs holding in queue $i$. For the sake of simplicity, we assume that if two jobs are being processed and one job finishes, then the other job can be preempted and both servers can be allocated to new jobs. For example, if both servers are working on jobs in queue 2 when a service is completed, then both servers can be immediately assigned to jobs in queue 1 . Our goal is to develop an optimal strategy that minimizes the expected total holding costs until the system is cleared. That is, the target is to minimize the expression

$$
\mathrm{E}\left[\int_{0}^{\infty}\left(c_{1} Q_{1}(t)+c_{2} Q_{2}(t)\right) \mathrm{d} t\right]
$$

Received 15 August 2002; revision received 25 January 2005.

* Postal address: Fachbereich Mathematik, Fachhochschule Wels, Stelzhamerstr. 23, Wels, A-4600, Austria.

Email address: k.schiefermayr@fh-wels.at

** Postal address: Institut für Stochastik, Universität Linz, Altenbergerstr. 69, Linz, A-4040, Austria.

Email address: josef.weichbold@jku.at 
where $Q_{1}(t)$ and $Q_{2}(t)$ denote the number of jobs at time $t$ in queue 1 and in queue 2, respectively. The starting point of our considerations was the paper of Ahn et al. [2], who considered this model with only one difference: in their model, if two jobs are being processed and one job finishes, then the other job is not preempted and only one server can be allocated to a new job. In [2], necessary and sufficient conditions on the parameters $c_{1}, c_{2}, \mu_{1}$, and $\mu_{2}$ were given for the optimality of allocating both servers to queue 1 or queue 2. More precisely, it was shown, by induction arguments, that the optimal strategy allocates both servers to queue 1 or to queue 2 (if possible) if and only if

$$
\frac{c_{1}}{c_{2}} \geq 1+\frac{\mu_{2}}{\mu_{1}} \quad \text { or } \quad \frac{c_{1}}{c_{2}} \leq 1+\frac{\mu_{2}}{\mu_{1}+\mu_{2}},
$$

respectively. In the case that

$$
1+\frac{\mu_{2}}{\mu_{1}+\mu_{2}}<\frac{c_{1}}{c_{2}}<1+\frac{\mu_{2}}{\mu_{1}}
$$

[2] gave a numerical example which suggests that, in this case, the optimal strategy is rather complicated.

Under the assumption of preempting the other job if one job is finished, we are able to give a complete description of the optimal strategy, especially for the case (1). Moreover, we will see that, in most cases, the optimal strategy for our model is easily transferable to the model considered in [2].

Other related works on tandem queues are [1], [3], [4], [5], [6], and [7]. Ahn et al. [1] considered a model with arrivals, two stations, and two flexible servers, and gave conditions under which it is optimal to allocate both servers to station 1 or 2 . In the model of [3], there is one server fixed to process type-1 jobs while the second server can process both types of jobs. In [5], Hajek considered a model with interacting service stations, where arrivals to the servers take place. His goal was to find the optimal allocation of the arriving customers to the different servers. Pandelis and Teneketzis [6] considered multiserver scheduling in a system with two interconnected queues, where jobs completing service in queue 1 join queue 2 with a certain probability. The processing time has a general distribution and they proved that, under certain conditions, a pure nonpreemptive strategy is optimal.

Farrar [4] also considered a two-stage tandem queueing system with a given initial number of customers and no further arrivals at any later time. In addition to there being one server at each station, there is an extra server that can be allocated to each station. The aim is to allocate the extra server in such a way that the expected total holding costs incurred until the system is empty are minimized. He showed that the optimal control policy is transition monotone.

The model of Rosberg et al. [7] consists of a Poisson arrival stream into a network of two $\mathrm{M} / \mathrm{M} / 1$ service stations in tandem, where the service rate at station 1 can be selected from an interval $u \in[0, a]$, as a function of the numbers of customers waiting at each station, in such a way as to minimize the expected total discounted or average cost. They showed that the optimal policy is of the form $u=0$ or $u=a$. An additional result is that, for the case of discounted cost, the optimal process can be nonergodic whereas the process in the case of average cost $i s$ ergodic.

The rest of the paper is organized as follows. In Section 2, we explain the model and introduce the optimal control matrix $(\mathcal{C}(i, j))_{i, j=0}^{\infty}$. With the help of this matrix, the optimal control can be illustrated graphically. The main results concerning the optimal control are given in Section 3. In Section 4, some structural lemmas are stated and proved. 
These will be needed for the proofs of the theorems for the control matrix, which are given in Section 5. Finally, in Section 6, the connection to [2] and some future work are discussed.

\section{The model}

Let $(i, j, X)$ denote the state of the system, where $i$ and $j$ are the numbers of jobs in queue 1 and queue 2 , respectively, and $X$ denotes the state of the two servers. There are four possible states for $X$, namely $11,22,12$, or $R$, where 11 means that we take two jobs from queue 1,22 means that we take two jobs from queue 2, 12 means that we take one job from each queue, and $R$ means that the server has finished processing a job and a decision must be made as to whether to choose 11,22 , or 12 . Furthermore, we define $V(i, j, X)$ to be the minimal expected total holding costs accumulated until the system is cleared. The function $V(i, j, X)$ satisfies the following dynamic equations:

$$
\begin{aligned}
V(i, j, 11)= & \frac{i c_{1}+j c_{2}}{2 \mu_{1}}+V(i-1, j+1, R), & i \geq 2, j \geq 0 \\
V(i, j, 22)= & \frac{i c_{1}+j c_{2}}{2 \mu_{2}}+V(i, j-1, R), & i \geq 0, j \geq 2 \\
V(i, j, 12)= & \frac{i c_{1}+j c_{2}}{\mu_{1}+\mu_{2}}+\frac{\mu_{1}}{\mu_{1}+\mu_{2}} V(i-1, j+1, R) & \\
& +\frac{\mu_{2}}{\mu_{1}+\mu_{2}} V(i, j-1, R), & i, j \geq 1
\end{aligned}
$$

and

$$
\begin{aligned}
V(i, j, R) & =\min \{V(i, j, 11), V(i, j, 22), V(i, j, 12)\}, & & i, j \geq 2, \\
V(i, 1, R) & =\min \{V(i, 1,11), V(i, 1,12)\}, & & i \geq 2, \\
V(1, j, R) & =\min \{V(1, j, 22), V(1, j, 12)\}, & & j \geq 2 .
\end{aligned}
$$

Furthermore, the following initial conditions are obviously valid:

$$
\begin{aligned}
V(1,1, R) & =V(1,1,12), \\
V(0, j, R) & =V(0, j, 22), \quad j \geq 2, \\
V(i, 0, R) & =V(i, 0,11), \quad i \geq 2, \\
V(1,0, R) & =\frac{c_{1}}{\mu_{1}}+\frac{c_{2}}{\mu_{2}}, \\
V(0,1, R) & =\frac{c_{2}}{\mu_{2}} .
\end{aligned}
$$

The following lemma states that, for $i, j \geq 2$, it is sufficient to consider only the two strategies $X=11$ and $X=22$, i.e. to take two jobs from queue 1 or two jobs from queue 2 .

Lemma 1. For $i, j \geq 2$,

$$
V(i, j, 12) \geq \min \{V(i, j, 11), V(i, j, 22)\},
$$

Proof. Let $i, j \geq 2$. Then the dynamic equations (2) give

$$
V(i, j, 12)=\frac{\mu_{1}}{\mu_{1}+\mu_{2}} V(i, j, 11)+\frac{\mu_{2}}{\mu_{1}+\mu_{2}} V(i, j, 22) .
$$

Thus, $V(i, j, 12)$ is a convex combination of $V(i, j, 11)$ and $V(i, j, 22)$, which gives (5). 


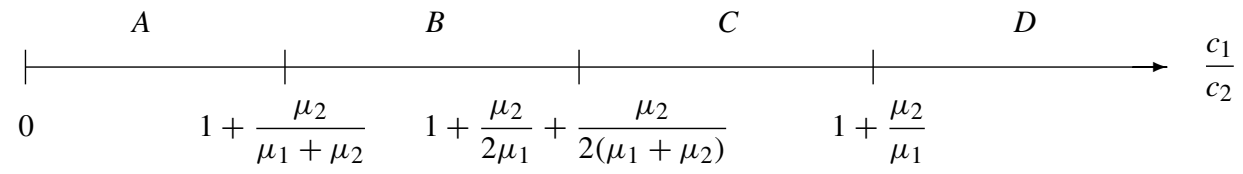

FIGURE 1: Parameter regions for $c_{1} / c_{2}$.

The optimal strategy is realized with the help of a control matrix $(\mathcal{C}(i, j))_{i, j=0}^{\infty}$ that, for each $(i, j) \in \mathbb{N}_{0} \times \mathbb{N}_{0}\left(\mathbb{N}_{0}=\{0,1,2, \ldots\}\right)$, gives the optimal control of the queue. Thus, if there are $i \geq 0$ jobs in queue 1 and $j \geq 0$ jobs in queue 2 , with $i+j \geq 2$, then we have at least one of the following three possibilities for control.

- Take two jobs of queue 1 , denoted by $\mathcal{C}(i, j)=11$.

- Take two jobs of queue 2 , denoted by $\mathcal{C}(i, j)=22$.

- Take one job of queue 1 and one job of queue 2 , denoted by $\mathcal{C}(i, j)=12$.

In order to obtain a unique control matrix, we define

$$
\begin{aligned}
& \mathcal{C}(i, j):=\left\{\begin{array}{ll}
11 & \text { if } V(i, j, 11) \leq V(i, j, 22), \\
22 & \text { if } V(i, j, 11)>V(i, j, 22),
\end{array} \quad i, j \geq 2,\right. \\
& \mathcal{C}(1, j):=\left\{\begin{array}{ll}
22 & \text { if } V(1, j, 22) \leq V(1, j, 12), \\
12 & \text { if } V(1, j, 22)>V(1, j, 12),
\end{array} \quad j \geq 2,\right. \\
& \mathcal{C}(i, 1):=\left\{\begin{array}{ll}
11 & \text { if } V(i, 1,11) \leq V(i, 1,12), \\
12 & \text { if } V(i, 1,11)>V(i, 1,12),
\end{array} \quad i \geq 2 .\right.
\end{aligned}
$$

Furthermore, by (4), we have

$$
\begin{array}{ll}
\mathcal{C}(i, 0) & =11, \quad i \geq 2, \\
\mathcal{C}(0, j) & =22, \quad j \geq 2, \\
\mathcal{C}(1,1) & =12 .
\end{array}
$$

Finally, for the sake of completeness, we define

$$
\begin{aligned}
& \mathcal{C}(0,0):=0, \\
& \mathcal{C}(1,0):=1, \\
& \mathcal{C}(0,1):=2 .
\end{aligned}
$$

In the next section, we give the control matrix $(\mathcal{C}(i, j))$, for all $(i, j) \in \mathbb{N}_{0} \times \mathbb{N}_{0}$, for all possible parameters $c_{1}, c_{2}, \mu_{1}$, and $\mu_{2}$. To this end, we separate the parameter region of $c_{1} / c_{2}$ into four subregions (see Figure 1):

$$
\begin{gathered}
A=\left(0,1+\frac{\mu_{2}}{\mu_{1}+\mu_{2}}\right], \quad B=\left(1+\frac{\mu_{2}}{\mu_{1}+\mu_{2}}, 1+\frac{\mu_{2}}{2 \mu_{1}}+\frac{\mu_{2}}{2\left(\mu_{1}+\mu_{2}\right)}\right), \\
C=\left[1+\frac{\mu_{2}}{2 \mu_{1}}+\frac{\mu_{2}}{2\left(\mu_{1}+\mu_{2}\right)}, 1+\frac{\mu_{2}}{\mu_{1}}\right), \quad D=\left[1+\frac{\mu_{2}}{\mu_{1}}, \infty\right) .
\end{gathered}
$$




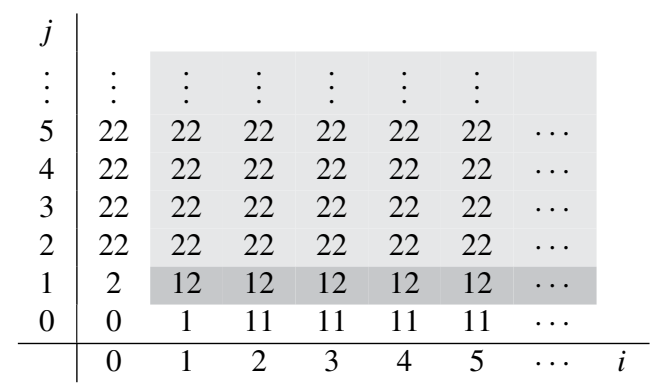

FIGURE 2: The control matrix for region $A$.

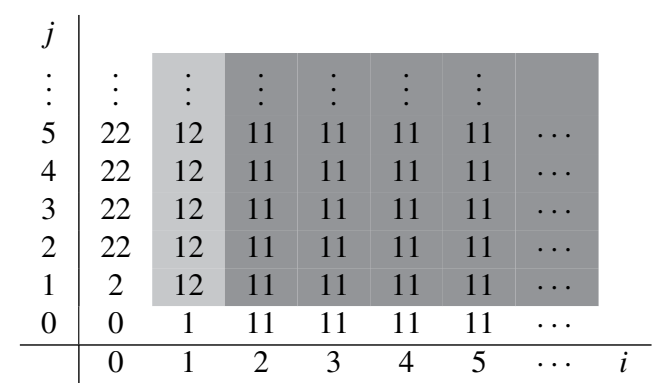

FIGURE 3: The control matrix for region $D$.

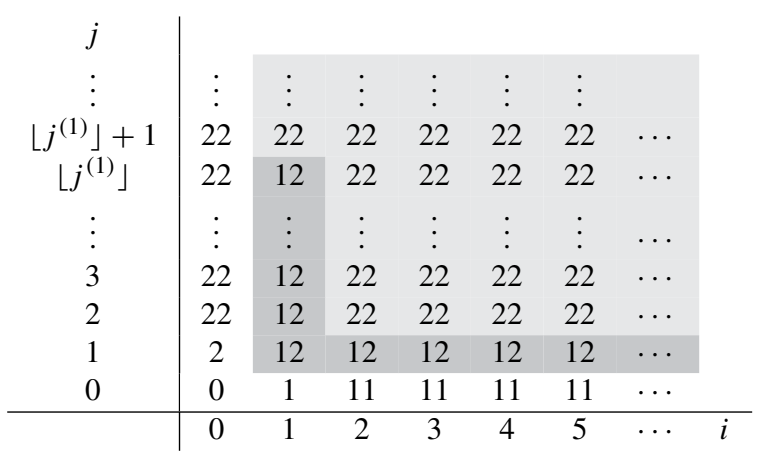

FIGURE 4: The control matrix for region $B$.

We will show that the control matrices for the above regions are of the following forms. (Recall that the optimal controls for $i=0, j \geq 0$ and $i \geq 0, j=1$ are obvious and will not be discussed.) For $c_{1} / c_{2} \in A$ or $c_{1} / c_{2} \in D$, it is always optimal (if possible) to allocate both servers to queue 2 or queue 1, respectively (see Figure 2 and Figure 3 ). For regions $B$ and $C$, the situation is more complicated. For $c_{1} / c_{2} \in B$ there is a partial column $(i=1)$ and a total row $(j=1)$ of $12 \mathrm{~s}$, and the other entries are $22 \mathrm{~s}$, while for $c_{1} / c_{2} \in C$ there is a triangular region of $11 \mathrm{~s}$, a partial column $(i=1)$ and a partial row $(j=1)$ of $12 \mathrm{~s}$, and the other entries are 22s (see Figure 4 and Figure 5, where $\lfloor\cdot\rfloor$ denotes the largest integer smaller than or equal to its argument). 


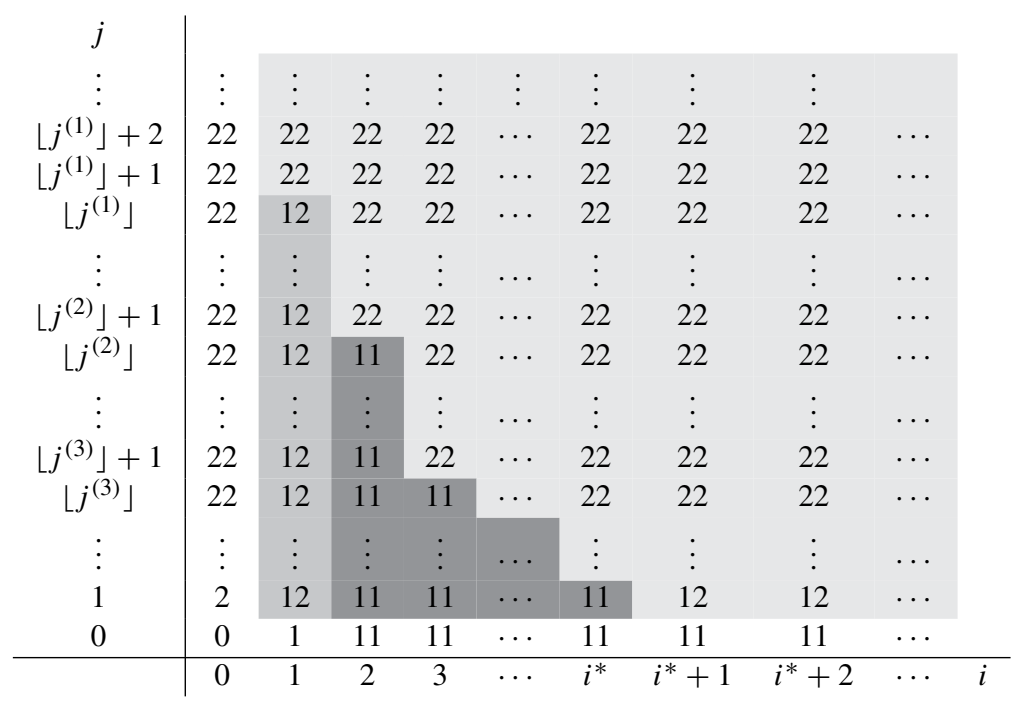

FIGURE 5: The control matrix for region $C$.

\section{Main results}

In this section, we present the main results concerning the optimal control of the system. For each parameter region $A, B, C$, and $D$, we explicitly give the control matrix $(\mathcal{C}(i, j))$. First we determine the control matrix (see Figures 2-5) for the entire parameter region for the case $i=1$, i.e. when there is one customer waiting at server 1 . Then we give the rest of the control matrices for the different regions.

Theorem 1. (a) For $c_{1} / c_{2} \in A$,

$$
\mathcal{C}(1, j)=22, \quad j \geq 2 .
$$

(b) For $c_{1} / c_{2} \in B \cup C$, there exists a $j^{(1)} \geq 2$ such that

$$
\mathcal{C}(1, j)=\left\{\begin{array}{l}
12 \quad \text { if } 2 \leq j<j^{(1)}, \\
22 \text { if } j \geq j^{(1)},
\end{array}\right.
$$

and $j^{(1)}$ is in fact given by

$$
j^{(1)}=\frac{\log \left(1-c_{1} \mu_{1} /\left[c_{2}\left(\mu_{1}+\mu_{2}\right)\right]\right)}{\log \left(\mu_{2} /\left(\mu_{1}+\mu_{2}\right)\right)} .
$$

(c) For $c_{1} / c_{2} \in D$,

$$
\mathcal{C}(1, j)=12, \quad j \geq 2 .
$$

Theorem 2. For $c_{1} / c_{2} \in A$,

$$
\begin{array}{ll}
\mathcal{C}(i, 1) & =12, \quad i \geq 2 \\
\mathcal{C}(i, j) & =22, \quad i \geq 1, j \geq 2 .
\end{array}
$$


Theorem 3. For $c_{1} / c_{2} \in B$,

$$
\begin{array}{ll}
\mathcal{C}(i, 1)=12, & i \geq 2, \\
\mathcal{C}(i, j)=22, & i \geq 2, \quad j \geq 2 .
\end{array}
$$

Theorem 4. Let $c_{1} / c_{2} \in C$. Then there exists an index $i^{*} \geq 2$ such that the following three assertions hold.

(a) For $j=1$,

$$
\mathcal{C}(i, 1)= \begin{cases}11 & \text { if } 2 \leq i \leq i^{*} \\ 12 & \text { if } i>i^{*}\end{cases}
$$

(b) There exist $j^{(2)}, \ldots, j^{\left(i^{*}\right)}$ such that, for $i \in\left\{2, \ldots, i^{*}\right\}$,

$$
\mathcal{C}(i, j)= \begin{cases}11 & \text { if } 1 \leq j \leq j^{(i)} \\ 22 & \text { if } j>j^{(i)}\end{cases}
$$

and $j^{(i)}$ is in fact given by

$$
j^{(i)}=-i+\frac{\log \left(-i \mu_{2}\left(c_{1} \mu_{1}-c_{2}\left(\mu_{1}+\mu_{2}\right)\right) /\left[c_{2}\left(\mu_{1}+\mu_{2}\right)^{2}\right]\right)}{\log \left(\mu_{2} /\left(\mu_{1}+\mu_{2}\right)\right)} .
$$

(c) For $i \geq i^{*}+1$ and $j \geq 2$,

$$
\mathcal{C}(i, j)=22 .
$$

Furthermore, the index $i^{*}$ is given by

$$
i^{*}=\max \{i \in \mathbb{N}: f(i) \leq 0\},
$$

where

$$
f(i):=1-\frac{1}{i}\left(\frac{\mu_{2}}{\mu_{1}+\mu_{2}}\right)^{i}-\frac{c_{1} \mu_{1}}{c_{2}\left(\mu_{1}+\mu_{2}\right)}
$$

is a strictly monotone-increasing function.

Remark 1. It is quite easy to see that

$$
j^{(1)}>j^{(2)}>\cdots>j^{\left(i^{*}\right)} \geq 1,
$$

where $j^{(1)}$ is given in (6). Inequality (10) is an immediate consequence of Lemma 3(a), below. Note also that, for $i=1$, formula (7) coincides with $j^{(1)}$ as given in (6). If, in Theorem 4(b), the number $j^{(i)}$ is an integer, then $V\left(i, j^{(i)}, 11\right)=V\left(i, j^{(i)}, 22\right)=V\left(i, j^{(i)}, 12\right)$.

Theorem 5. For $c_{1} / c_{2} \in D$,

$$
\mathcal{C}(i, j)=11, \quad i \geq 2, j \geq 1 \text {. }
$$




\section{Structural lemmas}

To proceed towards the proofs of our theorems, we must compute some costs and characterize the structure of our control matrices.

Lemma 2. (a) For $j \geq 1$,

$$
V(0, j, 22)=\frac{c_{2}\left(2+j+j^{2}\right)}{4 \mu_{2}} .
$$

(b) Let $j \geq 1$. If $\mathcal{C}(1, \ell)=12$ for $\ell \in\{1,2, \ldots, j\}$ then $V(1, j, 12)$ is given explicitly by

$$
\begin{gathered}
V(1, j, 12)=\frac{1}{4 \mu_{1}^{2} \mu_{2}}\left[4 c_{1} \mu_{1} \mu_{2}+c_{2}(\right. \\
\left(4+3 j+j^{2}\right) \mu_{1}^{2}-2 \mu_{2}^{2}\left(1-\left(\frac{\mu_{2}}{\mu_{1}+\mu_{2}}\right)^{j}\right) \\
\left.\left.+2 \mu_{1} \mu_{2}\left(j-1+\left(\frac{\mu_{2}}{\mu_{1}+\mu_{2}}\right)^{j}\right)\right)\right] .
\end{gathered}
$$

Proof. Part (a) is shown by induction on $j$. The cases $j=1$ and $j=2$ are clear. Assuming that (12) holds for $j$, we then have

$$
\begin{aligned}
V(0, j+1,22) & =\frac{(j+1) c_{2}}{2 \mu_{2}}+V(0, j, 22) \\
& =\frac{(j+1) c_{2}}{2 \mu_{2}}+\frac{c_{2}\left(2+j+j^{2}\right)}{4 \mu_{2}}=\frac{c_{2}\left(2+(j+1)+(j+1)^{2}\right)}{4 \mu_{2}},
\end{aligned}
$$

and the result follows.

Part (b) is also shown by induction on $j$. For $j=1$ we have

$$
\begin{aligned}
\frac{1}{4 \mu_{1}^{2} \mu_{2}} & {\left[4 c_{1} \mu_{1} \mu_{2}+c_{2}\left(8 \mu_{1}^{2}-2 \mu_{2}^{2}\left(1-\frac{\mu_{2}}{\mu_{1}+\mu_{2}}\right)+2 \mu_{1} \mu_{2} \frac{\mu_{2}}{\mu_{1}+\mu_{2}}\right)\right] } \\
= & \frac{1}{4 \mu_{1}^{2} \mu_{2}}\left[4 c_{1} \mu_{1} \mu_{2}+8 c_{2} \mu_{1}^{2}\right]=\frac{c_{1}}{\mu_{1}}+\frac{2 c_{2}}{\mu_{2}} \\
= & V(1,1,12) .
\end{aligned}
$$

Assuming that $\mathcal{C}(1, \ell)=12$ for $\ell \in\{1,2, \ldots, j\}$ and that (13) holds for $j$, we then have

$$
V(1, j+1,12)=\frac{c_{1}+(j+1) c_{2}}{\mu_{1}+\mu_{2}}+\frac{\mu_{1}}{\mu_{1}+\mu_{2}} V(0, j+2,22)+\frac{\mu_{2}}{\mu_{1}+\mu_{2}} V(1, j, 12) .
$$

By using the induction hypothesis and (12), we obtain

$$
\begin{aligned}
V(1, j+1,12)= & \frac{c_{1}+(j+1) c_{2}}{\mu_{1}+\mu_{2}}+\left(\frac{\mu_{1}}{\mu_{1}+\mu_{2}}\right) \frac{c_{2}\left(2+(j+2)+(j+2)^{2}\right)}{4 \mu_{2}} \\
& +\left(\frac{\mu_{2}}{\mu_{1}+\mu_{2}}\right) \frac{1}{4 \mu_{1}^{2} \mu_{2}}\left[4 c_{1} \mu_{1} \mu_{2}+c_{2}\left(\left(4+3 j+j^{2}\right) \mu_{1}^{2}\right.\right. \\
& \left.\left.+2 \mu_{2}^{2}\left(-1+\left(\frac{\mu_{2}}{\mu_{1}+\mu_{2}}\right)^{j}\right)+2 \mu_{1} \mu_{2}\left(-1+j+\left(\frac{\mu_{2}}{\mu_{1}+\mu_{2}}\right)^{j}\right)\right)\right]
\end{aligned}
$$


which, after some algebra, gives

$$
\begin{aligned}
V(1, j+1,12)=\frac{1}{4 \mu_{1}^{2} \mu_{2}} & {\left[4 c_{1} \mu_{1} \mu_{2}+c_{2} \mu_{1}^{2}\left(4+3(j+1)+(j+1)^{2}\right)\right.} \\
& +2 c_{2} \mu_{2}^{2}\left(-1+\left(\frac{\mu_{2}}{\mu_{1}+\mu_{2}}\right)^{j+1}\right) \\
& \left.+2 c_{2} \mu_{1} \mu_{2}\left(j+\left(\frac{\mu_{2}}{\mu_{1}+\mu_{2}}\right)^{j+1}\right)\right],
\end{aligned}
$$

as required.

In the next two lemmas, we give some structural results for the control matrix $(\mathcal{C}(i, j))_{i, j=0}^{\infty}$.

Lemma 3. Let $c_{1} / c_{2}<1+\mu_{2} / \mu_{1}$, i.e. $c_{1} / c_{2} \in A \cup B \cup C$.

(a) Let $i \geq 1$ and $j \geq 3$. If $\mathcal{C}(i, j)=22$ then $\mathcal{C}(i+1, j-1)=22$.

(b) Let $i \geq 1$. If $\mathcal{C}(i, 2)=22$ then $\mathcal{C}(i+1,1)=12$.

(c) Let $i \geq 3$ and $j \geq 1$. If $\mathcal{C}(i, j)=11$ then $\mathcal{C}(i-1, j+1)=11$.

(d) Let $j \geq 1$. If $\mathcal{C}(2, j)=11$ then $\mathcal{C}(1, j+1)=12$.

Proof. (a) We will compare the costs for the two different strategies in the state $(i+1, j-1)$. If we assume that $\mathcal{C}(i+1, j-1)=11$ then, by $\mathcal{C}(i, j)=22$, we get into the state $(i, j-1)$. On the other hand, if we assume that $\mathcal{C}(i+1, j-1)=22$ then we get into the state $(i+1, j-2)$. There we take the suboptimal strategy 11 (since, in this state, we do not know the optimal strategy) and again get into the state $(i, j-1)$. By showing that the costs along the second path are smaller than those along the first path, we will conclude that $\mathcal{C}(i+1, j-1)=22$.

Let $\mathcal{C}(i, j)=22$. Then, by (5), it suffices to show that

$$
V(i+1, j-1,22)-V(i+1, j-1,11)<0 .
$$

By the dynamic equations (2), and since $V(i+1, j-2, R) \leq V(i+1, j-2,11)$, we have

$$
\begin{aligned}
V(i+ & 1, j-1,22)-V(i+1, j-1,11) \\
= & \frac{(i+1) c_{1}+(j-1) c_{2}}{2 \mu_{2}}+V(i+1, j-2, R)-\frac{(i+1) c_{1}+(j-1) c_{2}}{2 \mu_{1}}-V(i, j, R) \\
\leq & \frac{(i+1) c_{1}+(j-1) c_{2}}{2 \mu_{2}}+V(i+1, j-2,11)-\frac{(i+1) c_{1}+(j-1) c_{2}}{2 \mu_{1}}-V(i, j, 22) \\
= & \frac{(i+1) c_{1}+(j-1) c_{2}}{2 \mu_{2}}+\frac{(i+1) c_{1}+(j-2) c_{2}}{2 \mu_{1}}+V(i, j-1, R) \\
& -\frac{(i+1) c_{1}+(j-1) c_{2}}{2 \mu_{1}}-\frac{i c_{1}+j c_{2}}{2 \mu_{2}}-V(i, j-1, R) \\
= & \frac{c_{1}}{2 \mu_{2}}-c_{2}\left(\frac{1}{2 \mu_{2}}+\frac{1}{2 \mu_{1}}\right)=\frac{c_{2}}{2 \mu_{1} \mu_{2}}\left[\frac{c_{1}}{c_{2}}-\left(1+\frac{\mu_{2}}{\mu_{1}}\right)\right] \\
< & 0 .
\end{aligned}
$$


(b) Let $\mathcal{C}(i, 2)=22$. Then, by (2), (3), and (4), we have

$$
\begin{aligned}
V(i+ & 1,1,12)-V(i+1,1,11) \\
= & \frac{(i+1) c_{1}+c_{2}}{\mu_{1}+\mu_{2}}+\frac{\mu_{1}}{\mu_{1}+\mu_{2}} V(i, 2, R)+\frac{\mu_{2}}{\mu_{1}+\mu_{2}} V(i+1,0, R) \\
& -\frac{(i+1) c_{1}+c_{2}}{2 \mu_{1}}-V(i, 2, R) \\
= & \frac{(i+1) c_{1}+c_{2}}{\mu_{1}+\mu_{2}}+\frac{\mu_{2}}{\mu_{1}+\mu_{2}} V(i+1,0,11)-\frac{(i+1) c_{1}+c_{2}}{2 \mu_{1}}-\frac{\mu_{2}}{\mu_{1}+\mu_{2}} V(i, 2,22) \\
= & \frac{(i+1) c_{1}+c_{2}}{\mu_{1}+\mu_{2}}+\frac{\mu_{2}}{\mu_{1}+\mu_{2}}\left(\frac{(i+1) c_{1}}{2 \mu_{1}}+V(i, 1, R)\right) \\
& -\frac{(i+1) c_{1}+c_{2}}{2 \mu_{1}}-\frac{\mu_{2}}{\mu_{1}+\mu_{2}}\left(\frac{i c_{1}+2 c_{2}}{2 \mu_{2}}+V(i, 1, R)\right) \\
= & \frac{c_{1} \mu_{1}-c_{2}\left(\mu_{1}+\mu_{2}\right)}{2 \mu_{1}\left(\mu_{1}+\mu_{2}\right)}=\frac{c_{2} \mu_{1}}{2 \mu_{1}\left(\mu_{1}+\mu_{2}\right)}\left[\frac{c_{1}}{c_{2}}-\left(1+\frac{\mu_{2}}{\mu_{1}}\right)\right] \\
< & 0 .
\end{aligned}
$$

(c) For $j=1$, part (c) is the contraposition of part (b) and, for $i \geq 3$ and $j \geq 2$, part (c) is the contraposition of part (a).

(d) This statement is the contraposition of part (a) for $i=1$.

Lemma 4. Let $j \geq 2$.

(a) Let

$$
1+\frac{\mu_{2}}{\mu_{1}+\mu_{2}}<\frac{c_{1}}{c_{2}}<1+\frac{\mu_{2}}{2\left(\mu_{1}+\mu_{2}\right)}+\frac{\mu_{2}}{2 \mu_{1}},
$$

i.e. $c_{1} / c_{2} \in B$. If

$$
\mathcal{C}(1, j)=\mathcal{C}(1, j+1)=12
$$

then $\mathcal{C}(2, j)=22$.

(b) Let

$$
1+\frac{\mu_{2}}{2\left(\mu_{1}+\mu_{2}\right)}+\frac{\mu_{2}}{2 \mu_{1}} \leq \frac{c_{1}}{c_{2}}<1+\frac{\mu_{2}}{\mu_{1}},
$$

i.e. $c_{1} / c_{2} \in C$, and let $j>j^{(2)}$, where $j^{(2)}$ is given by (7). If

$$
\mathcal{C}(1, j)=\mathcal{C}(1, j+1)=12
$$

then $\mathcal{C}(2, j)=22$.

(c) Let

$$
1+\frac{\mu_{2}}{2\left(\mu_{1}+\mu_{2}\right)}+\frac{\mu_{2}}{2 \mu_{1}} \leq \frac{c_{1}}{c_{2}}<1+\frac{\mu_{2}}{\mu_{1}},
$$

i.e. $c_{1} / c_{2} \in C$, let $i \geq 2$, and let $j>j^{(i+1)}$, where $j^{(i+1)}$ is given by (7). If

$$
\mathcal{C}(i, j)=\mathcal{C}(i, j+1)=11
$$

then $\mathcal{C}(i+1, j)=22$. 
Proof. Let $i \geq 1$ and $j \geq 2$. From Lemmas 3(c), 3(d), and 2(b), we obtain

$$
\begin{aligned}
V(i+ & 1, j, 22)-V(i+1, j, 11) \\
= & \frac{(i+1) c_{1}+j c_{2}}{2 \mu_{2}}+V(i+1, j-1, R)-V(i+1, j, 11) \\
\leq & \frac{(i+1) c_{1}+j c_{2}}{2 \mu_{2}}+V(i+1, j-1,11)-V(i+1, j, 11) \\
= & \frac{(i+1) c_{1}+j c_{2}}{2 \mu_{2}}+V(1, i+j-1,12)-V(1, i+j, 12)-\frac{i c_{2}}{2 \mu_{1}} \\
= & \frac{(i+1) c_{1}+j c_{2}}{2 \mu_{2}}+\frac{1}{2 \mu_{1} \mu_{2}} \\
& \times\left[c_{2}\left(\frac{\mu_{2}}{\mu_{1}+\mu_{2}}\right)^{i+j}\left(\mu_{1}+\mu_{2}\right)-c_{2}\left((1+i+j) \mu_{1}+\mu_{2}\right)\right]-\frac{i c_{2}}{2 \mu_{1}} \\
= & \frac{1}{2 \mu_{1} \mu_{2}}\left[c_{1}(i+1) \mu_{1}+c_{2}\left[\left(\frac{\mu_{2}}{\mu_{1}+\mu_{2}}\right)^{i+j}\left(\mu_{1}+\mu_{2}\right)-(i+1)\left(\mu_{1}+\mu_{2}\right)\right]\right] .
\end{aligned}
$$

If we set $i=1$ in (14) then we obtain

$$
\begin{aligned}
V(2, j, 22)-V(2, j, 11) & \leq \frac{c_{1}}{\mu_{2}}-\frac{c_{2}}{\mu_{2}}-\frac{c_{2}}{\mu_{1}}+\frac{c_{2}\left(\mu_{2} /\left(\mu_{1}+\mu_{2}\right)\right)^{j}}{2 \mu_{1}} \\
& <\frac{c_{1}}{\mu_{2}}-\frac{c_{2}}{\mu_{2}}-\frac{c_{2}}{\mu_{1}}+\frac{c_{2} \mu_{2}}{2 \mu_{1}\left(\mu_{1}+\mu_{2}\right)} \\
& =\frac{c_{2}}{\mu_{2}}\left[\frac{c_{1}}{c_{2}}-\left(1+\frac{\mu_{2}}{2 \mu_{1}}+\frac{\mu_{2}}{2\left(\mu_{1}+\mu_{2}\right)}\right)\right] \\
& <0,
\end{aligned}
$$

which gives the assertion of part (a).

In cases (b) and (c), by (14), $V(i+1, j, 22)-V(i+1, j, 11)<0$ is satisfied if

$$
c_{2}\left(\frac{\mu_{2}}{\mu_{1}+\mu_{2}}\right)^{i+j+1} \frac{\left(\mu_{1}+\mu_{2}\right)^{2}}{\mu_{2}}<-(i+1)\left(c_{1} \mu_{1}-c_{2}\left(\mu_{1}+\mu_{2}\right)\right),
$$

which is equivalent to

$$
j>-(i+1)+\frac{\log \left(-(i+1) \mu_{2}\left(c_{1} \mu_{1}-c_{2}\left(\mu_{1}+\mu_{2}\right)\right) /\left[c_{2}\left(\mu_{1}+\mu_{2}\right)^{2}\right]\right)}{\log \left(\mu_{2} /\left(\mu_{1}+\mu_{2}\right)\right)}=j^{(i+1)} .
$$

For $i=1$, this gives part (b), and for $i \geq 2$, it gives part (c). This completes the proof.

\section{Lemma 5.}

$$
\mathcal{C}(2,1)=\left\{\begin{array}{l}
11 \text { if } \frac{c_{1}}{c_{2}} \geq 1+\frac{\mu_{2}}{2\left(\mu_{1}+\mu_{2}\right)}+\frac{\mu_{2}}{2 \mu_{1}}, \text { i.e. } \frac{c_{1}}{c_{2}} \in C \cup D, \\
12 \text { if } \frac{c_{1}}{c_{2}}<1+\frac{\mu_{2}}{2\left(\mu_{1}+\mu_{2}\right)}+\frac{\mu_{2}}{2 \mu_{1}}, \text { i.e. } \frac{c_{1}}{c_{2}} \in A \cup B .
\end{array}\right.
$$


Proof. By Lemma 3(d) and Lemma 2(b),

$$
\begin{aligned}
V(2, & 1,11)-V(2,1,12) \\
& =\frac{2 c_{1}+c_{2}}{2 \mu_{1}}+V(1,2,12)-\frac{2 c_{1}+c_{2}}{\mu_{1}+\mu_{2}}-\frac{\mu_{1}}{\mu_{1}+\mu_{2}} V(1,2,12)-\frac{\mu_{2}}{\mu_{1}+\mu_{2}} V(2,0,11) \\
& =\frac{2 c_{1}+c_{2}}{2 \mu_{1}}-\frac{2 c_{1}+c_{2}}{\mu_{1}+\mu_{2}}-\frac{\mu_{2}}{\mu_{1}+\mu_{2}} \frac{2 c_{1}}{2 \mu_{1}}+\frac{\mu_{2}}{\mu_{1}+\mu_{2}}(V(1,2,12)-V(1,1,12)) \\
& =\frac{2 c_{1}+c_{2}}{2 \mu_{1}}-\frac{2 c_{1}+c_{2}}{\mu_{1}+\mu_{2}}-\frac{\mu_{2}}{\mu_{1}+\mu_{2}} \frac{2 c_{1}}{2 \mu_{1}}+\frac{\mu_{2}}{\mu_{1}+\mu_{2}}\left(\frac{3 c_{2}}{2 \mu_{2}}+\frac{c_{2}}{2\left(\mu_{1}+\mu_{2}\right)}\right) \\
& =\frac{1}{2 \mu_{1} \mu_{2}\left(\mu_{1}+\mu_{2}\right)}\left(-2 \mu_{1} \mu_{2} c_{1}+c_{2}\left(2 \mu_{1} \mu_{2}+\mu_{2}^{2}+\frac{\mu_{1} \mu_{2}^{2}}{\mu_{1}+\mu_{2}}\right)\right) \\
& =\frac{c_{2}}{\mu_{1}+\mu_{2}}\left(1+\frac{\mu_{2}}{2 \mu_{1}}+\frac{\mu_{2}}{2\left(\mu_{1}+\mu_{2}\right)}-\frac{c_{1}}{c_{2}}\right)
\end{aligned}
$$

which gives the assertion.

Remark 2. Note that, for

$$
\frac{c_{1}}{c_{2}}=1+\frac{\mu_{2}}{2\left(\mu_{1}+\mu_{2}\right)}+\frac{\mu_{2}}{2 \mu_{1}},
$$

we have $V(2,1,11)=V(2,1,12)$.

\section{Proofs of the theorems}

\subsection{Proof of Theorem 1}

First note that $\mathcal{C}(1,1)=12$. Define

$$
j^{*}:=\sup \{j: \mathcal{C}(1, \ell)=12,1 \leq \ell \leq j\} .
$$

A simple computation gives

$$
V(1,2,12)-V(1,2,22)=\frac{c_{2}}{2 \mu_{2}}\left(1+\frac{\mu_{2}}{\mu_{1}+\mu_{2}}-\frac{c_{1}}{c_{2}}\right),
$$

whence

$$
\mathcal{C}(1,2)= \begin{cases}12 & \text { if } \frac{c_{1}}{c_{2}}>1+\frac{\mu_{2}}{\mu_{1}+\mu_{2}}, \text { i.e. } \frac{c_{1}}{c_{2}} \in B \cup C \cup D, \\ 22 & \text { if } \frac{c_{1}}{c_{2}} \leq 1+\frac{\mu_{2}}{\mu_{1}+\mu_{2}}, \text { i.e. } \frac{c_{1}}{c_{2}} \in A\end{cases}
$$

Thus, for

$$
\frac{c_{1}}{c_{2}} \leq 1+\frac{\mu_{2}}{\mu_{1}+\mu_{2}}
$$

i.e. $c_{1} / c_{2} \in A$, we have $j^{*}=1$.

Now let

$$
\frac{c_{1}}{c_{2}}>1+\frac{\mu_{2}}{\mu_{1}+\mu_{2}}
$$


i.e. $c_{1} / c_{2} \in B \cup C \cup D$. Using Lemma 2(b), we find, for all $j, 1 \leq j \leq j^{*}$, that

$$
\begin{aligned}
V(1, j, 12)-V(1, j, 22) \\
\quad=V(1, j, 12)-\frac{c_{1}+j c_{2}}{2 \mu_{2}}-V(1, j-1,12) \\
\quad=\frac{1}{2 \mu_{1} \mu_{2}}\left[c_{2}\left(\mu_{1}+j \mu_{1}+\mu_{2}-\left(\frac{\mu_{2}}{\mu_{1}+\mu_{2}}\right)^{j}\left(\mu_{1}+\mu_{2}\right)\right)\right]-\frac{c_{1}+j c_{2}}{2 \mu_{2}} .
\end{aligned}
$$

Thus, $V(1, j, 12)-V(1, j, 22)<0$ is equivalent to

$$
-\left(\frac{\mu_{2}}{\mu_{1}+\mu_{2}}\right)^{j}\left(1+\frac{\mu_{2}}{\mu_{1}}\right)<\frac{c_{1}}{c_{2}}-\left(1+\frac{\mu_{2}}{\mu_{1}}\right) .
$$

Consequently, if

$$
\frac{c_{1}}{c_{2}} \geq 1+\frac{\mu_{2}}{\mu_{1}},
$$

i.e. $c_{1} / c_{2} \in D$, then the right-hand side of (15) is nonnegative. Since the left-hand side of (15) is negative, we have $V(1, j, 12)<V(1, j, 22)$; hence, $\mathcal{C}(1, j)=12$ for every $j \geq 2$ and, in this case, $j^{*}=\infty$. This proves part (c).

If, on the other hand,

$$
1+\frac{\mu_{2}}{\mu_{1}+\mu_{2}}<\frac{c_{1}}{c_{2}}<1+\frac{\mu_{2}}{\mu_{1}}
$$

i.e. $c_{1} / c_{2} \in B \cup C$, then (15) is equivalent to

$$
j<\frac{\log \left(1-c_{1} \mu_{1} /\left[c_{2}\left(\mu_{1}+\mu_{2}\right)\right]\right)}{\log \left(\mu_{2} /\left(\mu_{1}+\mu_{2}\right)\right)} .
$$

Hence, we have proved that there exists a $j^{(1)} \geq 2$ such that $\mathcal{C}(1, j)=12$, for $2 \leq j<j^{(1)}$, and $\mathcal{C}\left(1,\left\lfloor j^{(1)}\right\rfloor+1\right)=22$, where $j^{(1)}$ is given by (6) and, recall, $\lfloor x\rfloor$ denotes the largest integer smaller than or equal to $x$. Thus, (6) and the stated values of the optimal control $\mathcal{C}(1, j), j<j^{(1)}$, have been verified.

Finally, let

$$
\frac{c_{1}}{c_{2}}<1+\frac{\mu_{2}}{\mu_{1}},
$$

i.e. $c_{1} / c_{2} \in A \cup B \cup C$. We will prove case (a) and the remainder of case (b) by induction on $j$. Note that we have already proved that $\mathcal{C}(1,2)=22$ in case (a) and

$$
\mathcal{C}\left(1,\left\lfloor j^{(1)}\right\rfloor+1\right)=22
$$

in case (b); this is our basis for induction.

Assume that $\mathcal{C}(1, j)=22$. Then, from

$$
\begin{aligned}
0 \geq & V(1, j, 22)-V(1, j, 12) \\
= & \frac{c_{1}+j c_{2}}{2 \mu_{2}}+V(1, j-1, R)-\frac{c_{1}+j c_{2}}{\mu_{1}+\mu_{2}}-\frac{\mu_{1}}{\mu_{1}+\mu_{2}} V(0, j+1,22) \\
& -\frac{\mu_{2}}{\mu_{1}+\mu_{2}} V(1, j-1, R)
\end{aligned}
$$


we find the inequality

$$
\frac{\mu_{1}}{\mu_{1}+\mu_{2}} V(1, j-1, R) \leq \frac{c_{1}+j c_{2}}{\mu_{1}+\mu_{2}}+\frac{\mu_{1}}{\mu_{1}+\mu_{2}} V(0, j+1,22)-\frac{c_{1}+j c_{2}}{2 \mu_{2}} .
$$

Thus, by the induction hypothesis $V(1, j, R)=V(1, j, 22)$ and (16), we obtain

$$
\begin{aligned}
V(1, j+ & 1,22)-V(1, j+1,12) \\
= & \frac{c_{1}+(j+1) c_{2}}{2 \mu_{2}}+V(1, j, R)-\frac{c_{1}+(j+1) c_{2}}{\mu_{1}+\mu_{2}}-\frac{\mu_{1}}{\mu_{1}+\mu_{2}} V(0, j+2,22) \\
& -\frac{\mu_{2}}{\mu_{1}+\mu_{2}} V(1, j, R) \\
= & \frac{c_{1}+(j+1) c_{2}}{2 \mu_{2}}+\frac{\mu_{1}}{\mu_{1}+\mu_{2}} V(1, j, 22)-\frac{c_{1}+(j+1) c_{2}}{\mu_{1}+\mu_{2}}-\frac{\mu_{1}}{\mu_{1}+\mu_{2}} V(0, j+2,22) \\
= & \frac{c_{1}+(j+1) c_{2}}{2 \mu_{2}}+\frac{\mu_{1}}{\mu_{1}+\mu_{2}}\left(\frac{c_{1}+j c_{2}}{2 \mu_{2}}+V(1, j-1, R)\right)-\frac{c_{1}+(j+1) c_{2}}{\mu_{1}+\mu_{2}} \\
& -\frac{\mu_{1}}{\mu_{1}+\mu_{2}} V(0, j+2,22) \\
= & \frac{c_{1}+(j+1) c_{2}}{2 \mu_{2}}+\frac{\mu_{1}}{\mu_{1}+\mu_{2}} \frac{c_{1}+j c_{2}}{2 \mu_{2}}+\frac{\mu_{1}}{\mu_{1}+\mu_{2}} V(1, j-1, R) \\
& -\frac{c_{1}+(j+1) c_{2}}{\mu_{1}+\mu_{2}}-\frac{\mu_{1}}{\mu_{1}+\mu_{2}} \frac{(j+2) c_{2}}{2 \mu_{2}}-\frac{\mu_{1}}{\mu_{1}+\mu_{2}} V(0, j+1,22) \\
\leq & \frac{c_{1}+(j+1) c_{2}}{2 \mu_{2}}+\frac{\mu_{1}}{\mu_{1}+\mu_{2}} \frac{c_{1}+j c_{2}}{2 \mu_{2}}+\frac{c_{1}+j c_{2}}{\mu_{1}+\mu_{2}}+\frac{\mu_{1}}{\mu_{1}+\mu_{2}} V(0, j+1,22) \\
& -\frac{c_{1}+j c_{2}}{2 \mu_{2}}-\frac{c_{1}+(j+1) c_{2}}{\mu_{1}+\mu_{2}}-\frac{\mu_{1}}{\mu_{1}+\mu_{2}} \frac{(j+2) c_{2}}{2 \mu_{2}}-\frac{\mu_{1}}{\mu_{1}+\mu_{2}} V(0, j+1,22) \\
= & \frac{c_{2}}{2 \mu_{2}}+\left(\frac{\mu_{2} \mu_{1}}{\mu_{1}+\mu_{2}}\right) \frac{c_{1}}{2 \mu_{2}}-\frac{c_{2}}{\mu_{1}+\mu_{2}}-\left(\frac{\mu_{1}}{\mu_{1}+\mu_{2}}\right) \frac{c_{2}}{\mu_{2}} \\
c_{2} & \left.\left(1+\frac{\mu_{2}}{\mu_{1}}\right)\right)<0 .
\end{aligned}
$$

Hence, $\mathcal{C}(1, j+1)=22$ and parts (a) and (b) follow, in their entireties, by induction.

\subsection{Proof of Theorem 2}

By Theorem 1(a) and Lemmas 3(a) and 3(b), the assertion follows immediately.

\subsection{Proof of Theorem 3}

By Lemma 5, we have $\mathcal{C}(2,1)=12$ in region $B$. Therefore, by Theorem $1(\mathrm{~b})$ and Lemmas 3(a), 3(b), and 4(a), the assertion follows immediately.

\subsection{Proof of Theorem 4}

Define

$$
i^{*}:=\sup \{i: \mathcal{C}(\ell, 1)=11,2 \leq \ell \leq i\}
$$


Since $\mathcal{C}(2,1)=11$ in region $C$ (see Lemma 5), we have $i^{*} \geq 2$ and, by Theorem 1 and Lemmas 3(a) and 3(b), we have $i^{*}<\infty$. Let $i \in\left\{2, \ldots, i^{*}\right\}$ be fixed and define $j^{*}$ by

$$
\begin{aligned}
j^{*} & =\sup \{j: \mathcal{C}(i, \ell)=11,1 \leq \ell \leq j\} \\
& =\sup \{j: V(i, j, 11)-V(i, j, 22) \leq 0,1 \leq \ell \leq j\}
\end{aligned}
$$

By Theorem 1 and Lemma 3(a), the index $j^{*}$ is finite. Our goal is to compute a formula for $j^{(i)}$. By Lemmas 2(b), 3(c), and 3(d), for every $j \in\left\{2, \ldots, j^{*}\right\}$, we have

$$
\begin{aligned}
V(i, j, 11)-V(i, j, 22) \\
\quad=V(i, j, 11)-\frac{i c_{1}+j c_{2}}{2 \mu_{2}}-V(i, j-1,11) \\
\quad=V(1, i+j-1,12)-V(1, i+j-2,12)+\frac{(i-1) c_{2}}{2 \mu_{1}}-\frac{i c_{1}+j c_{2}}{2 \mu_{2}} \\
=\frac{c_{2} \mu_{2}\left((i+j) \mu_{1}+\mu_{2}\right)-c_{2}\left(\mu_{2} /\left(\mu_{1}+\mu_{2}\right)\right)^{i+j}\left(\mu_{1}+\mu_{2}\right)^{2}}{2 \mu_{1} \mu_{2}^{2}}+\frac{(i-1) c_{2}}{2 \mu_{1}}-\frac{i c_{1}+j c_{2}}{2 \mu_{2}} \\
=i c_{2}\left(\frac{1}{2 \mu_{1}}+\frac{1}{2 \mu_{2}}\right)-\frac{i c_{1}}{2 \mu_{2}}-\frac{c_{2}\left(\mu_{2} /\left(\mu_{1}+\mu_{2}\right)\right)^{i+j}\left(\mu_{1}+\mu_{2}\right)^{2}}{2 \mu_{1} \mu_{2}^{2}} .
\end{aligned}
$$

Thus, $V(i, j, 11)-V(i, j, 22) \leq 0$ is equivalent to

$$
-c_{2}\left(\frac{\mu_{2}}{\mu_{1}+\mu_{2}}\right)^{i+j}\left(\mu_{1}+\mu_{2}\right)^{2} \leq-i \mu_{2}\left(c_{2}\left(\mu_{1}+\mu_{2}\right)-c_{1} \mu_{1}\right),
$$

which is in turn equivalent to

$$
j \leq-i+\frac{\log \left(-i \mu_{2}\left(c_{1} \mu_{1}-c_{2}\left(\mu_{1}+\mu_{2}\right)\right) /\left[c_{2}\left(\mu_{1}+\mu_{2}\right)^{2}\right]\right)}{\log \left(\mu_{2} /\left(\mu_{1}+\mu_{2}\right)\right)} .
$$

It follows that

$$
\begin{aligned}
\mathcal{C}(i, j) & =11, \\
\mathcal{C}\left(i,\left\lfloor j^{(i)}\right\rfloor+1\right) & =22,
\end{aligned}
$$

for $i \in\left\{2, \ldots, i^{*}\right\}$ and $1 \leq j \leq j^{(i)}$, with $j^{(i)}$ as given in (7). By Lemmas 4(b), 4(c), and 3(a), it follows that $\mathcal{C}(i, j)=22$ for all $i=1, \ldots, i^{*}$ and $j>j^{(i)}$. Hence, part (b) has been entirely proved.

We next consider the column $i=i^{*}+1$. By the definition of $i^{*}$ and $j^{(i)}$, we have $j^{\left(i^{*}+1\right)}<1$. Thus, by Lemmas 4(c) and 3(a), we find that

$$
\mathcal{C}\left(i^{*}+1, j\right)=22 \text { for all } j \geq 2
$$

Hence, by (17) and Lemma 3(a), part (c) immediately follows. By part (c) and Lemma 3(b), we then have $\mathcal{C}(i, 1)=12$ for $i \geq i^{*}+1$. This proves part (a) in its entirety.

Now we prove (8) and (9). With the help of Lemmas 3(c) and 3(d), we can easily compute the difference $V(i, 1,11)-V(i, 1,12)$. Indeed, using Lemma 2(b), for all $i \in\left\{2, \ldots, i^{*}\right\}$ 
we obtain

$$
\begin{aligned}
& V(i, 1,11)-V(i, 1,12) \\
&=\frac{i c_{1}+c_{2}}{2 \mu_{1}}+V(i-1,2,11)-\frac{i c_{1}+c_{2}}{\mu_{1}+\mu_{2}}-\frac{\mu_{1}}{\mu_{1}+\mu_{2}} V(i-1,2,11)-\frac{\mu_{2}}{\mu_{1}+\mu_{2}} V(i, 0,11) \\
&= \frac{i c_{1}+c_{2}}{2 \mu_{1}}-\frac{i c_{1}+c_{2}}{\mu_{1}+\mu_{2}}-\frac{i c_{1} \mu_{2}}{2 \mu_{1}\left(\mu_{1}+\mu_{2}\right)}+\frac{\mu_{2}}{\mu_{1}+\mu_{2}}(V(i-1,2,11)-V(i-1,1,11)) \\
&= \frac{i c_{1}+c_{2}}{2 \mu_{1}}-\frac{i c_{1}+c_{2}}{\mu_{1}+\mu_{2}}-\frac{i c_{1} \mu_{2}}{2 \mu_{1}\left(\mu_{1}+\mu_{2}\right)} \\
&+\frac{\mu_{2}}{\mu_{1}+\mu_{2}}\left(\frac{(i-2) c_{2}}{2 \mu_{1}}+V(1, i, 12)-V(1, i-1,12)\right) \\
&= \frac{i c_{1}+c_{2}}{2 \mu_{1}}-\frac{i c_{1}+c_{2}}{\mu_{1}+\mu_{2}}-\frac{i c_{1} \mu_{2}}{2 \mu_{1}\left(\mu_{1}+\mu_{2}\right)} \\
&+\frac{\mu_{2}}{\mu_{1}+\mu_{2}}\left(\frac{(i-2) c_{2}}{2 \mu_{1}}+\frac{c_{2}\left(\mu_{1}+i \mu_{1}+\mu_{2}-\left(\mu_{2} /\left(\mu_{1}+\mu_{2}\right)\right)^{i}\left(\mu_{1}+\mu_{2}\right)\right)}{2 \mu_{1} \mu_{2}}\right) \\
&= \frac{1}{2 \mu_{1} \mu_{2}\left(\mu_{1}+\mu_{2}\right)}\left[-c_{1} i \mu_{1} \mu_{2}+c_{2}\left(i \mu_{2}\left(\mu_{1}+\mu_{2}\right)-\mu_{2}\left(\mu_{1}+\mu_{2}\right)\left(\frac{\mu_{2}}{\mu_{1}+\mu_{2}}\right)\right)\right] \\
&= \frac{c_{2} i}{2 \mu_{1}}\left(-\frac{c_{1} \mu_{1}}{c_{2}\left(\mu_{1}+\mu_{2}\right)}+1-\frac{1}{i}\left(\frac{\mu_{2}}{\mu_{1}+\mu_{2}}\right)^{i}\right) .
\end{aligned}
$$

Thus, having $V(i, 1,11)-V(i, 1,12) \leq 0$ is equivalent to having $f(i) \leq 0$, where $f(i)$ is as defined in (9). We conclude that $i^{*}=\max \{i \in \mathbb{N}: f(i) \leq 0\}$, since $f(i)$ is strictly monotone increasing in $i$, .

\subsection{Proof of Theorem 5}

Let $i \geq 2$. We will prove (11) by induction on $j$. For $j=1$, we have

$$
\begin{aligned}
& V(i, 1,11)-V(i, 1,12) \\
&=\frac{i c_{1}+c_{2}}{2 \mu_{1}}+V(i-1,2, R)-\frac{i c_{1}+c_{2}}{\mu_{1}+\mu_{2}}-\frac{\mu_{1}}{\mu_{1}+\mu_{2}} V(i-1,2, R)-\frac{\mu_{2}}{\mu_{1}+\mu_{2}} V(i, 0,11) \\
&=\frac{i c_{1}+c_{2}}{2 \mu_{1}}+\frac{\mu_{2}}{\mu_{1}+\mu_{2}} V(i-1,2, R)-\frac{i c_{1}+c_{2}}{\mu_{1}+\mu_{2}}-\frac{\mu_{2}}{\mu_{1}+\mu_{2}}\left(\frac{i c_{1}}{2 \mu_{1}}+V(i-1,1, R)\right) \\
& \leq \frac{i c_{1}+c_{2}}{2 \mu_{1}}+\frac{\mu_{2}}{\mu_{1}+\mu_{2}} V(i-1,2,22)-\frac{i c_{1}+c_{2}}{\mu_{1}+\mu_{2}}-\frac{\mu_{2}}{\mu_{1}+\mu_{2}}\left(\frac{i c_{1}}{2 \mu_{1}}+V(i-1,1, R)\right) \\
&= \frac{i c_{1}+c_{2}}{2 \mu_{1}}+\frac{\mu_{2}}{\mu_{1}+\mu_{2}}\left(\frac{(i-1) c_{1}+2 c_{2}}{2 \mu_{2}}+V(i-1,1, R)\right) \\
&-\frac{i c_{1}+c_{2}}{\mu_{1}+\mu_{2}}-\frac{\mu_{2}}{\mu_{1}+\mu_{2}}\left(\frac{i c_{1}}{2 \mu_{1}}+V(i-1,1, R)\right) \\
&= \frac{1}{2 \mu_{1} \mu_{2}\left(\mu_{1}+\mu_{2}\right)}\left[c_{1}\left(i\left(\mu_{1}+\mu_{2}\right) \mu_{2}-2 i \mu_{1} \mu_{2}+(i-1) \mu_{1} \mu_{2}-i \mu_{2}^{2}\right)\right. \\
&= \frac{c_{2}}{\mu_{1}+\mu_{2}}\left(1+\frac{\mu_{2}}{\mu_{1}}-\frac{c_{1}}{c_{2}}\right) \leq 0 .
\end{aligned}
$$


Hence, we have proved that $\mathcal{C}(i, 1)=11$ for $i \geq 2$. Now assume that $\mathcal{C}(i, j)=11$. Then

$$
\begin{aligned}
& V(i, j+1,11)-V(i, j+1,22) \\
&= \frac{i c_{1}+(j+1) c_{2}}{2 \mu_{1}}+V(i-1, j+2, R)-\frac{i c_{1}+(j+1) c_{2}}{2 \mu_{2}}-V(i, j, 11) \\
& \leq \frac{i c_{1}+(j+1) c_{2}}{2 \mu_{1}}+V(i-1, j+2,22)-\frac{i c_{1}+(j+1) c_{2}}{2 \mu_{2}}-V(i, j, 11) \\
&= \frac{i c_{1}+(j+1) c_{2}}{2 \mu_{1}}+\frac{(i-1) c_{1}+(j+2) c_{2}}{2 \mu_{2}}+V(i-1, j+1, R) \\
&-\frac{i c_{1}+(j+1) c_{2}}{2 \mu_{2}}-\frac{i c_{1}+j c_{2}}{2 \mu_{1}}-V(i-1, j+1, R) \\
&= c_{2}\left(\frac{1}{2 \mu_{1}}+\frac{1}{2 \mu_{2}}\right)-\frac{c_{1}}{2 \mu_{2}}=\frac{c_{2}}{2 \mu_{2}}\left[\left(1+\frac{\mu_{2}}{\mu_{1}}\right)-\frac{c_{1}}{c_{2}}\right] \\
& \leq 0 .
\end{aligned}
$$

Hence, the induction process is complete and Theorem 5 follows in its entirety.

\section{Discussion}

\subsection{The relation between the preemptive and the nonpreemptive models}

In this section, we discuss the differences between our results and those obtained in [2]. The main difference between the two models, as mentioned in the introduction, is that we preempt the servers when one job finishes. Therefore, in each state $(i, j)$, we can allocate both servers to the queues, as if we had started in this state. This preemptive strategy makes it possible to calculate the optimal control for all regions $A, B, C$, and $D$. In the following, we will call our model the preemptive model and the one in [2] the nonpreemptive model. Due the memoryless property of the exponential distribution, in the preemptive model the costs in each state $(i, j)$, until the system is cleared, are less than or equal to those in the nonpreemptive model. Hence, if it is possible, in each state $(i, j)$ in the nonpreemptive case, to satisfy the optimal control of the preemptive model along the path taken until the system is empty, then the control optimal in the preemptive model is also optimal in the nonpreemptive one. Therefore, we obtain the following corollary for the nonpreemptive model.

Corollary 1. (a) Let $c_{1} / c_{2} \in A \cup B \cup D$. Then the control matrix of the nonpreemptive model is the same as that of the preemptive model.

(b) Let $c_{1} / c_{2} \in C$ and $(i, j) \in \mathbb{N} \times \mathbb{N}$. If either $i=1$ or $\mathcal{C}(i, j)=11$, then the control $\mathcal{C}(i, j)$ optimal in the preemptive model is also optimal in the nonpreemptive model.

Thus, for regions $A, B$, and $D$, and partially for region $C$, our control matrix is also optimal in the nonpreemptive model. Of course, for regions $A$ and $D$, this is a rederivation of the results of [2] but, due to our structural lemmas, by a shorter method.

Now let $c_{1} / c_{2} \in C$. For those $(i, j) \in \mathbb{N} \times \mathbb{N}$ for which $\mathcal{C}(i, j) \neq 11$, the optimal control in the nonpreemptive case is more complicated, so let us explain the differences between the preemptive and nonpreemptive models in an example. Let $\mu_{1}=\mu_{2}=1, c_{1}=1.995$, and $c_{2}=1$, i.e. $c_{1} / c_{2} \in C$. Figures 6 and 7 show the control matrices in the preemptive and nonpreemptive cases, respectively. Note that, in the nonpreemptive case, the control matrix only gives the optimal control in $(i, j)$ in the beginning. It is not clear what happens if we start 


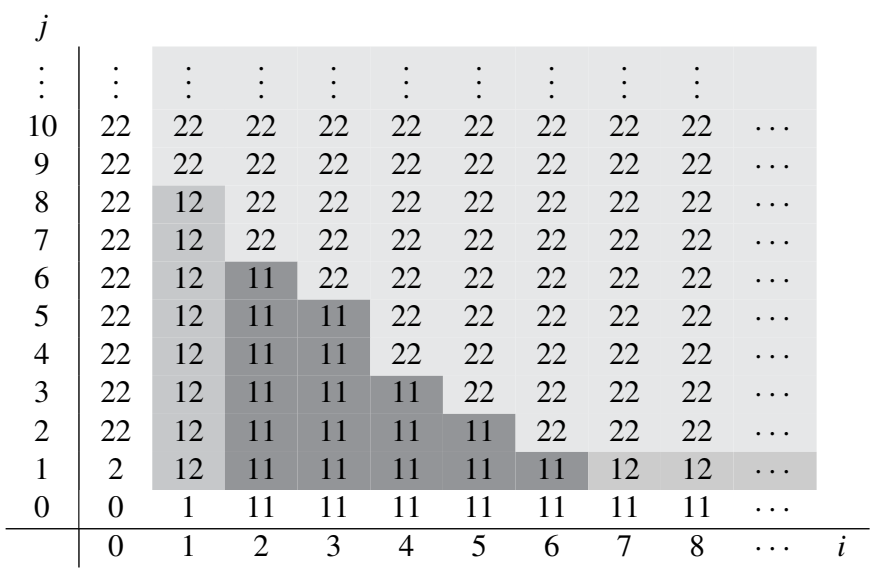

FIGURE 6: The control matrix in the preemptive case for the parameters $\mu_{1}=\mu_{2}=1, c_{1}=1.995$, and $c_{2}=1$.

\begin{tabular}{|c|c|c|c|c|c|c|c|c|c|c|}
\hline : & $\vdots$ & $\vdots$ & $\vdots$ & $\vdots$ & • & $\vdots$ & $\vdots$ & $\vdots$ & $\vdots$ & \\
\hline 10 & 22 & 22 & 22 & 22 & 22 & 22 & 22 & 22 & 22 & $\ldots$ \\
\hline 9 & 22 & 22 & 22 & 22 & 22 & 22 & 22 & 22 & 22 & $\ldots$ \\
\hline 8 & 22 & 12 & 22 & 22 & 22 & 22 & 22 & 22 & 22 & $\ldots$ \\
\hline 7 & 22 & 12 & 12 & 22 & 22 & 22 & 22 & 22 & 22 & $\ldots$ \\
\hline 6 & 22 & 12 & 11 & 22 & 22 & 22 & 22 & 22 & 22 & $\ldots$ \\
\hline 5 & 22 & 12 & 11 & 11 & 22 & 22 & 22 & 22 & 22 & $\ldots$ \\
\hline 4 & 22 & 12 & 11 & 11 & 12 & 22 & 22 & 22 & 22 & $\ldots$ \\
\hline 3 & 22 & 12 & 11 & 11 & 11 & 12 & 22 & 22 & 22 & $\ldots$ \\
\hline 2 & 22 & 12 & 11 & 11 & 11 & 11 & 12 & 22 & 22 & $\cdots$ \\
\hline 1 & 2 & 12 & 11 & 11 & 11 & 11 & 11 & 12 & 12 & $\ldots$ \\
\hline \multirow[t]{2}{*}{0} & 0 & 1 & 11 & 11 & 11 & 11 & 11 & 11 & 11 & $\ldots$ \\
\hline & 0 & 1 & 2 & 3 & 4 & 5 & 6 & 7 & 8 & $\cdots$ \\
\hline
\end{tabular}

FIGURE 7: The control matrix in [2] (the nonpreemptive case) for the parameters $\mu_{1}=\mu_{2}=1$, $c_{1}=1.995$, and $c_{2}=1$.

in $(i, j)=(3,6)$ and get into the state $(3,5)$, because there the optimal control 11 cannot be realized. By comparing the figures, we also see that the first columns (i.e. $i=1)$ and the states with optimal control 11 are identical, as stated in Theorem 1(b). In Figure 7, $\mathcal{C}(2,7)=12$ and $\mathcal{C}(3,6)=22$; therefore, from the preemptive strategy we cannot conclude what is optimal at the positions $\left(i,\left\lfloor j^{(i)}\right\rfloor+1\right), i \geq 2$, in the nonpreemptive strategy. The rest of the control matrix seems to be identical in both strategies.

\subsection{The case of one server}

Since we did not find the case with only one server in the literature, for completeness we here state the optimal control for the one-server problem. The proof is easy and, therefore, omitted. 
Theorem 6. In the case of one server, it is always optimal to serve a job of queue 1 or queие 2 (if possible) if and only if

$$
\frac{c_{1}}{c_{2}} \geq 1+\frac{\mu_{2}}{\mu_{1}} \quad \text { or } \quad \frac{c_{1}}{c_{2}}<1+\frac{\mu_{2}}{\mu_{1}}
$$

respectively.

\subsection{Extensions}

From these results there arise some interesting questions.

- How does the control matrix change if there are arrivals to the queues?

- What is the optimal control for a tandem queue with two servers, where the served jobs of queue 1 join queue 2 with a probability $p$ and leave the system with probability $1-p$ ? This problem will be solved in a forthcoming paper [8], which will describe the connection between the case $p=1$ and the case $p=0$. The latter case has been extensively investigated in the literature.

- What is the optimal control if there are more than two servers?

\section{Acknowledgements}

We would like to thank P. Weiß and C. Takacs for many discussions on the subject.

\section{References}

[1] Ahn, H., Duenyas, I. And Lewis, M. E. (2002). Optimal control of a two-stage tandem queuing system with flexible servers. Prob. Eng. Inf. Sci. 16, 453-469.

[2] Ahn, H., Duenyas, I. And Zhang, R. Q. (1999). Optimal stochastic scheduling of a two-stage tandem queue with parallel servers. Adv. Appl. Prob. 31, 1095-1117.

[3] Ahn, H., Duenyas, I. and Zhang, R. Q. (2004). Optimal control of a flexible server. Adv. Appl. Prob. 36, 139-170.

[4] Farrar, T. M. (1993). Optimal use of an extra server in a two station tandem queueing network. IEEE Trans. Automatic Control 38, 1296-1299.

[5] HAJEK, B. (1984). Optimal control of two interacting service stations. IEEE Trans. Automatic Control 29, 491-499.

[6] Pandelis, D. G. And Teneketzis, D. (1994). Optimal multiserver stochastic scheduling of two interconnected priority queues. Adv. Appl. Prob. 26, 258-279.

[7] Rosberg, Z., Varaiya, P. P. and Walrand, J. C. (1982). Optimal control of service in tandem queues. IEEE Trans. Automatic Control 27, 600-610.

[8] Schiefermayr, K. And Weichbold, J. (2005). The optimal control of a general tandem queue. To appear in Prob. Eng. Inf. Sci. 